\title{
SUBSTRATOS E TEMPERATURAS NA GERMINAÇÃO DE Ceiba speciosa E IDENTIFICAÇÃO DE FUNGOS POTENCIALMENTE PATOGÊNICOS
}

\author{
SENEME, Adriana Martinelli ${ }^{1}$ \\ FERIANI, Aurea Portes ${ }^{2}$ \\ MENDONÇA, Cristina Goncalves de ${ }^{2}$
}

RESUMO: Ceiba speciosa (A. St.-Hil.) Ravenna (Malvaceae) é uma espécie arbórea nativa, indicada para arborização urbana e recuperação de áreas degradadas, especialmente na mata ciliar. O comportamento germinativo e a qualidade sanitária das sementes de espécies florestais são características importantes para a produção de mudas. Assim, o presente trabalho teve como objetivos verificar temperaturas e substratos favoráveis à germinação e identificar os microrganismos potencialmente patogênicos que podem influenciar o desempenho fisiológico de sementes de $C$. speciosa. As sementes foram submetidas aos seguintes procedimentos: a) semeadura para avaliação da germinação: em substratos papel-filtro, areia fina e vermiculita em germinadores (B.O.D.) regulados para regimes de temperatura constante de $25^{\circ} \mathrm{C}, 30^{\circ} \mathrm{C}$ e alternado $25-30{ }^{\circ} \mathrm{C}$ (fotoperíodo de 8 horas); b) primeira contagem (PCG) com verificação de emissão da raiz primária (maior que $2 \mathrm{~mm}$ ); c) peso de matéria seca de plântula; d) emergência em casa de vegetação: foram utilizados os substratos areia fina e vermiculita (granulometria fina e média); e) teste de sanidade ("Blotter test"): foram utilizadas 400 sementes pelo método de papel-filtro. Após esse período, os fungos foram identificados com auxílio de estereoscópio. O delineamento experimental foi inteiramente casualizado com quatro repetições por tratamento e as médias foram comparadas pelo teste de Tukey $(\mathrm{P}>0,05 \%)$. Concluiu-se que a temperatura de $30^{\circ} \mathrm{C}$ e os substratos areia e vermiculita foram os mais favoráveis à germinação das sementes, com porcentagens superiores a 75\%. Os fungos identificados foram Aspergillus sp. (incidência 1\%), Rhizoctonia solani (1\%), Alternaria sp. (1\%), Cladosporium sp. (1\%), Fusarium sp. (5\%), Phytium sp. (1\%), Rhizopus stolonifer (2\%) e Curvularia sp. (1\%).

Palavras-Chave: Espécie florestal. Fungos patogênicos. Malvaceae.

\begin{abstract}
SUMMARY: Ceiba speciosa is a tree and native species suitable for urban forestation and recovery areas, especially riparian forest. Studies on the germinating behavior and sanitary quality of seeds in forest species are important silvicultural aspects for the production of seedlings. Thus, this study aimed to verify temperatures and substrates favorable for seeds germination and identify potentially pathogenic microorganisms that can influence its physiological performance. The seeds were submitted to the following: a) germination: substrates filter paper, sand and vermiculite in germination (BOD) adjusted to constant temperature regimes of $25^{\circ} \mathrm{C}, 30^{\circ} \mathrm{C}$ and alternate $25-30 \mathrm{C}$ (photoperiod 8 hours); b) first count (PCG) with verification of primary root emission (greater than $2 \mathrm{~mm}$ ); c) dry weight of seedling; d) emergence in the greenhouse: the substrates sand and vermiculite (fine and medium) were used; e) sanity check ("Blotter test"): used to seed 400 by the filter paper method. After this period, the fungi were identified under estereomicroscope. O experimental design was completely randomized with four replications and the means were compared by Tukey test $(\mathrm{P}>0.05 \%)$. It was concluded that the $30^{\circ} \mathrm{C}$ temperature and the substrates sand and vermiculite were the most favorable for seed germination, with percentages above $75 \%$. The fungi were identified Aspergillus sp. (incidence 1\%), Rhizoctonia solani (1\%), Alternaria sp. (1\%), Cladosporium sp (1\%), Fusarium sp. (5\%), Pythium sp. (1\%), Rhizopus stolonifer (2\%) and Curvularia sp (1\%).
\end{abstract}

Keywords: Species forest. Pathogenic fungi. Malvaceae.

\section{INTRODUÇÃO}

Várias espécies no Brasil são denominadas popularmente como paineiras, porém a mais conhecida é a Ceiba speciosa (A.St.-Hill.) Ravenna (Malvaceae), nativa em florestas brasileiras.

É uma árvore indicada para arborização de parques, praças, jardins, avenidas e também de

\footnotetext{
${ }^{1}$ Professora Dra Adjunto III - Universidade Federal do Paraná - Departamento de Fitotecnia e Fitossanitarismo.

${ }^{2}$ Docente - Universidade Federal do Paraná- Departamento Fitotecnia e Fitossanitarismo
} 
rodovias. Possui grande efeito ornamental devido ao seu porte e beleza das flores. No Brasil, distribui-se naturalmente, principalmente, nos estados de Rio de Janeiro, Minas Gerais, Goiás, São Paulo, Mato Grosso do Sul e Paraná, ocorrendo em floresta latifoliada semidecídua, tanto em matas primárias como secundárias (BORGES et al., 1998).

A espécie é recomendada para plantios para reconstituição de matas e recuperação de mata ciliar em locais sem inundação. Segundo Carvalho, Silva e Davide. (2006), a paineira é uma espécie clímax, exigente de luz e suas sementes são ortodoxas, ou seja, podem ser armazenadas por períodos longos sob baixa temperatura e umidade relativa. As sementes não apresentam dormência, porém a imersão em água fria por $2 \mathrm{~h}$ facilita a germinação (CARVALHO, 2003). Possui taxa de germinação superior a $80 \%$ e emergência muito rápida, entre 5 e 8 dias. $\mathrm{O}$ desenvolvimento das plântulas no campo é rápido, com crescimento de 5 a $6 \mathrm{~m}$ em dois anos (LORENZI, 1992). As sementes apresentam comportamento variável frente à temperatura. A faixa de $20-30{ }^{\circ} \mathrm{C}$ mostra-se adequada para a germinação de grande número de espécies tropicais e subtropicais (BORGES; RENA, 1993). Segundo revisão de literatura realizada por Brancalion, Novembre e Rofrigues (2010), a temperatura de $25^{\circ} \mathrm{C}$ é considerada ótima para a maioria das espécies arbóreas dos biomas Cerrado e Mata Atlântica seguida de $30^{\circ} \mathrm{C}$ para as do bioma Amazônia.

Muitos organismos fitopatogênicos podem ser transportados pelas sementes, porém a transmissão de vários deles por esse meio não é totalmente conhecida (ARAÚJO, 2008); as consequências desse transporte podem ser a introdução do patógeno em áreas isentas, o aumento do inóculo na área e a redução da qualidade fisiológica das sementes (MACHADO, 1988; MENTEN, 1991). Também pode ocorrer morte da semente antes da emergência ou destruição das plântulas recém-emergidas (tombamento de pósemergência) (LINK; COSTA, 1982).

Há carência de informações sobre sementes florestais nativas, tanto em relação à qualidade sanitária como qualidade fisiológica e condições de germinação. Na literatura existem alguns trabalhos sobre germinação e caracterização fisiológica de sementes de paineira (FANTI; PEREZ, 2005; GUEDES; ALVES, 2011; LEMES; LOPES, 2012) e algumas informações sobre sua qualidade sanitária, embora ainda escassas e que necessitam de maiores investigações.

A qualidade das sementes não se expressa somente em seu valor genético e estado físico e fisiológico, mas também no aspecto sanitário, o qual refere-se, em princípio, à presença ou ausência de agentes patogênicos (principalmente os fungos). A condição sanitária é extremamente importante se consideramos que as sementes são veículos de agentes fitopatogênicos, que nelas podem se alojar e com elas serem levados ao campo, provocando redução na germinação e vigor e originando focos primários de infecção (GOULART, 2004).

Lazarotto, Muniz e Santos (2010) encontraram 13 gêneros de fungos em sementes de paineira, sendo cinco deles considerados potencialmente patogênicos (Alternaria, Curvularia, Pestalotia, Colletrotrichum e Fusarium). Fusarium sp. foi o fungo de maior incidência, o qual foi encontrado em todas as amostras e em ambos os métodos do teste de sanidade (BDA e papel de filtro). Em outro trabalho com sementes de paineira armazenadas, independente do período avaliado, foram detectados fungos dos gêneros Colletotrichum sp.; Aspergillus sp.; Penicillium sp.; Alternaria sp.; Fusarium sp. e a incidência de Aspergillus sp e Penicillium sp foi maior à medida que que houve aumento no período de armazenamento (SILVA et al., 2003). Fiumaro (2010) encontrou sete gêneros de fungos em sementes de paineira sendo o Fusarium de maior incidência (46,5\%), no entanto, a germinação não foi afetada. Em outras espécies florestais também foram encontrados patógenos em sementes (FERREIRA,1989; MARTINELLI-SENEME et al.,2006 et al., 2006; OLIVEIRA et al., 2012; MEDEIROS et al., 2015).

O trabalho teve como objetivos verificar temperaturas e substratos favoráveis à germinação e identificar os microrganismos potencialmente patogênicos ao desempenho fisiológico das 
sementes de C. speciosa.

\section{MATERIAL E MÉTODO}

O trabalho foi desenvolvido na Universidade Federal do Paraná (UFPR), no Departamento de Fitotecnia e Fitossanitarismo, em Curitiba. Sementes de C. speciosa foram provenientes do Instituto de Pesquisas Florestais de Piracicaba (IPEF) com teor de água inicial de 11\%, sendo submetidas aos seguintes procedimentos: a) semeadura para avaliação da germinação: em caixas plásticas transparentes com tampa (11 x $11 \times 3 \mathrm{~cm}$ ) utilizando-se os substratos papel filtro (duas folhas), areia (fina e peneirada, malha de $1,7 \mathrm{~mm}$, esterilizada por $24 \mathrm{~h}$ a $120^{\circ} \mathrm{C}$ ) e vermiculita (textura média, granulometria $2-4 \mathrm{~mm} \mathrm{e}$ densidade de $80-100 \mathrm{~g} / \mathrm{l}$, esterilizada por $24 \mathrm{~h}$ a $120{ }^{\circ} \mathrm{C}$ ). Os substratos areia e vermiculita foram umedecidos com água em $60 \%$ de sua capacidade de retenção e o papel 3,0 vezes seu peso (GUEDES; ALVES, 2012), sem umedecimento posterior. O teste de germinação foi conduzido em germinadores (B.O.D.) regulados para regimes de temperatura constante de $25^{\circ} \mathrm{C}$ e $30^{\circ} \mathrm{C}$ e também alternado de $25-30$ ${ }^{\circ} \mathrm{C}$ (fotoperíodo de $8 \mathrm{~h}$ ) e teve duração de 12 dias (GUEDES; ALVES, 2012). Na avaliação do teste de germinação, no $5^{\circ}$ dia após a instalação do teste, procedeu-se a primeira contagem (PCG) com verificação de emissão da raiz primária (maior que $2 \mathrm{~mm}$ ) (MARTINELLI-SENEME et al., 2006) que foi considerado um teste de vigor. Foram consideradas para o cálculo da porcentagem de germinação aos 12 dias todas as plântulas que apresentavam raiz primária maior que $2 \mathrm{~mm}$ (FANTI; PEREZ, 2004) ; b) peso de matéria seca de plântula: após o encerramento do teste de germinação, as plântulas foram colocadas em estufa de secagem na temperatura de $65^{\circ} \mathrm{C}$ durante quatro dias (NAKAGAWA, 1994) e os resultados expressos em gramas por plântula; c) emergência em casa de vegetação: foram utilizados substrato areia (fina e peneirada, malha de $1,7 \mathrm{~mm}$, esterilizada por $24 \mathrm{~h}$ a $120^{\circ} \mathrm{C}$ ) vermiculita fina (granulometria 1-2 mm e densidade de 90-120 g/l) e vermiculita média granulometria 2-4 mm e densidade de 80-100 g/l). O teste foi encerrado aos 10 dias quando cessou a emergência das plântulas e os resultados foram expressos em porcentagem; d) teste de sanidade ("Blotter test"): utilizaram-se 400 sementes pelo método de papel-filtro, que consistiu na semeadura em caixas tipo "gerbox" e incubação durante oito dias em ambiente de sala (temperatura em torno de $25^{\circ} \mathrm{C}$ ) previamente esterilizada sob luz constante. Após esse período, os fungos potencialmente patogênicos foram identificados com auxílio de microscópio estereoscópico e expressos em porcentagem.

O delineamento experimental foi inteiramente casualizado (DIC) com quatro repetições para cada tratamento e as médias foram comparadas pelo teste de Tukey $(\mathrm{P}>0,05 \%)$.

\section{RESULTADO E DISCUSSÃO}

Os resultados referentes à emissão de raiz primária, germinação e peso de matéria seca estão na Tabela 1.

Não houve diferença para substratos nas temperaturas constantes, porém em $20-30{ }^{\circ} \mathrm{C}$ o substrato papel proporcionou desempenho inferior na emissão da raiz primária. Os substratos areia e vermiculita, na temperatura de $25^{\circ} \mathrm{C}$, apresentaram desempenho superior em comparação à temperatura de $30^{\circ} \mathrm{C}$. Para o substrato papel o desempenho inferior também foi verificado na temperatura de 20-30 ${ }^{\circ} \mathrm{C}$. Considerando a temperatura como um fator isolado, constatou-se que os maiores valores de PCG foram obtidos em $25{ }^{\circ} \mathrm{C}$ e para o substrato vermiculita, em $25^{\circ} \mathrm{C}$, a diferença foi significativa em relação às demais temperaturas testadas.

O substrato papel proporcionou os menores valores de germinação nas temperaturas de $30^{\circ} \mathrm{Ce}$ 
20-30 ${ }^{\circ} \mathrm{C}$ com diferença significativa em relação aos demais substratos testados. Sementes nos substratos areia e vermiculita apresentaram o mesmo desempenho nas temperaturas de $30{ }^{\circ} \mathrm{C}$ e $20-30{ }^{\circ} \mathrm{C}$; em $25^{\circ} \mathrm{C}$ o substrato vermiculita foi o melhor, proporcionando $84 \%$ de germinação, maior média entre os testados. Nas três temperaturas avaliadas, o substrato papel proporcionou o pior desenvolvimento de plântulas. Os substratos não influenciaram a emergência das plântulas na casa de vegetação. Os valores de emergência foram menores do que aqueles observados no teste de germinação (Figura 1).

Tabela 1. Germinação (GE), emissão da raiz primária (ERP) e peso de matéria seca (PMS) de sementes de Ceiba speciosa em diferentes temperaturas $\left(25^{\circ} \mathrm{C}, 30^{\circ} \mathrm{C}\right.$ e $20-30{ }^{\circ} \mathrm{C}$ ) e substratos (areia, vermiculita e papel).

\begin{tabular}{|c|c|c|c|}
\hline \multirow[t]{2}{*}{ GE (\%) Substrato } & \multicolumn{3}{|c|}{ Temperatura } \\
\hline & $25^{\circ} \mathrm{C}$ & $30{ }^{\circ} \mathrm{C}$ & $20-30{ }^{\circ} \mathrm{C}$ \\
\hline Areia & $71 \mathrm{bA}^{*}$ & $75 \mathrm{aA}$ & $75 \mathrm{aA}$ \\
\hline Vermiculita & $84 \mathrm{aA}$ & $78 \mathrm{aA}$ & $81 \mathrm{aA}$ \\
\hline Papel & $58 \mathrm{bA}$ & $56 \mathrm{bB}$ & $56 \mathrm{bB}$ \\
\hline \multicolumn{4}{|c|}{ Coeficiente de variação $(\%)=15,45$} \\
\hline \multirow[t]{2}{*}{ ERP (\%) Substrato } & \multicolumn{3}{|c|}{ Temperatura } \\
\hline & $25^{\circ} \mathrm{C}$ & $30{ }^{\circ} \mathrm{C}$ & $20-30{ }^{\circ} \mathrm{C}$ \\
\hline Areia & $92 \mathrm{aA}^{*}$ & $63 \mathrm{aB}$ & $81 \mathrm{aA}$ \\
\hline Vermiculita & $91 \mathrm{aA}$ & $61 \mathrm{aB}$ & $67 \mathrm{abB}$ \\
\hline Papel & $87 \mathrm{aA}$ & $75 \mathrm{aA}$ & $53 \mathrm{bB}$ \\
\hline \multicolumn{4}{|c|}{ Coeficiente de variação $(\%)=17,90$} \\
\hline \multirow[t]{2}{*}{ PMS (g) Substrato } & \multicolumn{3}{|c|}{$\begin{array}{ll}\text { Temperatura } \\
\end{array}$} \\
\hline & $25^{\circ} \mathrm{C}$ & $30^{\circ} \mathrm{C}$ & $20-30{ }^{\circ} \mathrm{C}$ \\
\hline Areia & $0,742 \mathrm{aB}^{*}$ & 1,121 aA & $1,150 \mathrm{aB}$ \\
\hline Vermiculita & $0,725 \mathrm{aB}$ & $1,085 \mathrm{aA}$ & $0,976 \mathrm{aA}$ \\
\hline Papel & $0,586 \mathrm{bA}$ & $0,300 \mathrm{bB}$ & $0,345 \mathrm{bB}$ \\
\hline
\end{tabular}

*Médias seguidas da mesma letra minúscula, na coluna, e maiúscula, na linha, não diferem a $5 \%$ de probabilidade de acordo com Tukey (5\%).

De acordo com os resultados observou-se, de maneira geral, que a temperatura de proporcionou maior velocidade de geminação, porém maiores valores de peso de matéria seca e de germinação final foram obtidos em $30^{\circ} \mathrm{C}$. O substrato papel apresentou o pior desempenho quando comparado à areia e vermiculita, no entanto, na emergência de plântulas em casa de vegetação não houve influência do substrato.

Figura 1. Emergência (\%) de plântulas de Ceiba speciosa nos substratos areia, vermiculita fina e vermiculita média em casa de vegetação (Tukey ns).

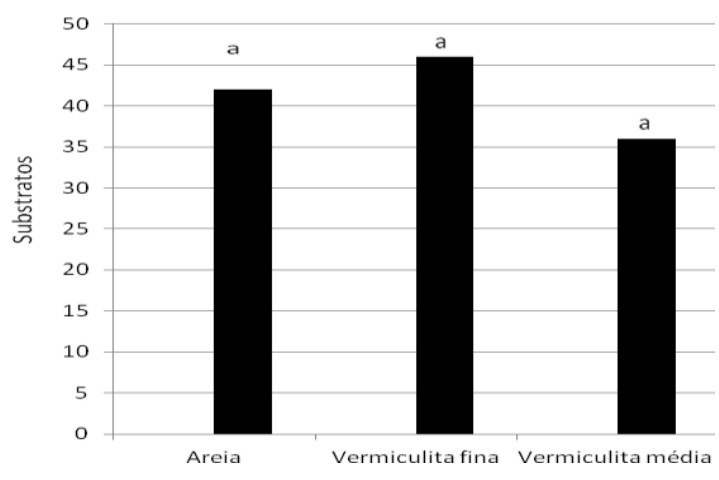


Guedes e Alves (2012) observaram que sementes de Ceiba glaziovii tiveram desempenho inferior na primeira contagem da germinação nos substratos sobre papel e papel toalha na temperatura $20-30{ }^{\circ} \mathrm{C}$. Em trabalho com sementes de ipê, Martins, Martinelli-Seneme e Nakagawa (2008) também verificaram que o substrato papel proporcionou pior desempenho na primeira contagem e na germinação quando comparado com o substrato areia. Martinelli-Seneme et al. (2008) observaram, também em sementes de ipê, que o substrato vermiculita proporcionou germinação mais rápida que o papel. $\mathrm{O}$ substrato papel proporciona menor área de contato com a semente, quando comparado aos demais, não favorecendo a embebição.

Os resultados de PCG concordam com Guedes e Alves (2011) que observaram melhor desempenho na primeira contagem de germinação na temperatura de $25{ }^{\circ} \mathrm{C}$ para C. glaziovii. Lemes e Lopes (2012) constataram que sementes de C. speciosa apresentaram maior velocidade de germinação e menor tempo médio de germinação também em $25^{\circ} \mathrm{C}$. Guedes e Alves (2011) observaram os menores valores de germinação nas temperaturas de $30{ }^{\circ} \mathrm{C}$ e $20-30{ }^{\circ} \mathrm{C}$ para sementes de C. glaziovii em teste de germinação sobre papel. Guedes e Alves (2012) verificaram que a areia foi o melhor substrato entre os avaliados para sementes de $C$. glaziovii. Os autores ainda recomendaram que o teste de germinação de $C$. glaziovii deve ser conduzido com substrato areia ou papel toalha. No presente experimento, para sementes de $C$. speciosa, os melhores resultados foram obtidos em areia e vermiculita.

Os patógenos encontrados nas sementes de C. speciosa foram Aspergillus sp. (1\%), Rhizoctonia solani (1\%), Alternaria sp. (1\%), Cladosporium sp. (1\%), Fusarium sp. (5\%), Phytium sp. (1\%), Rhizopus stolonifer (2\%) e Curvularia sp. (1\%). A incidência de patógenos nas sementes de paineira são bem menores que àqueles observados por Fiumaro (2010) que trabalhou com a mesma espécie. $\mathrm{O}$ autor encontrou sete gêneros de fungos fitopatogênicos, sendo o Fusarium o de maior incidência (46,5\%), no entanto, a germinação não foi afetada (81,5\%). Silva et al. (2003) observaram que sementes de $C$. speciosa em armazenamento (até 120 dias) independente do período, apresentaram fungos dos gêneros Colletotrichum sp.; Aspergillus sp.; Penicillium sp.; Alternaria sp.; Fusarium sp. Os autores verificaram que a incidência de Aspergillus, Colletotrichum e Penicillium aumentou durante o período de armazenagem e recomendam o tratamento das sementes armazenadas para favorecer a sanidade.

Em trabalho conduzido com lotes de sementes de C. speciosa de diversas procedências, Lazarotto, Muniz e Santos (2010) verificaram a presença dos gêneros Alternaria, Curvularia, Pestalotia, Colletrotrichum e Fusarium sendo que o gênero Fusarium também apresentou as maiores porcentagens de incidência tanto pelo método de papel de filtro quanto BDA. Segundo Ferreira (1989), há relatos de algumas espécies de Fusarium causando tombamento em pré ou pós-emergência de plântulas de espécies florestais, sendo problema comum em sementes. Em sementes de acácia (MEDEIROS et al., 2015) foram encontrados patógenos dos gêneros Aspergillus, Rhizopus, Penicillium e Nigrospora sendo que o menor valor encontrado foi para Rhizopus stolonifer com incidência de $17 \%$.

\section{CONCLUSÃO}

A temperatura $30^{\circ} \mathrm{C}$ e os substratos areia e vermiculita foram os mais favoráveis à germinação das sementes de C. speciosa, com porcentagens superiores a $75 \%$.

Nas sementes de C. speciosa foram identificados Aspergillus sp. (incidência de 1\%), Rhizoctonia solani (1\%), Alternaria sp. (1\%), Cladosporium sp. (1\%), Fusarium sp. (5\%), Phytium sp. (1\%), Rhizopus stolonifer (2\%) e Curvularia sp. (1\%). 


\section{REFERENCIAS}

ARAÚJO, E. R. Qualidade fisiológica, etiologia e patogenicidade de fungos assinalados em sementes de aroeira produzidas em três municípios da Paraíba. 2008. Areia: Universidade Federal da Paraíba, 2008.45p.

BORGES, E.E.L.; RENA, A.B. Germinação de sementes. In: AGUIAR, I.B.; PINÑA-RODRIGUES, F.C.M. ; FIGLIOLA, M.B. Sementes florestais tropicais. Brasília: ABRATES, 1993. p.83-136.

BORGES, L.C.et al. Disponibilidade de recursos oferecidos por paineiras - Chorisia speciosa - em ambientes urbanos. In: http://www.fevale.edu.br/seminario/cd/files/pdf/1798.pdf. 1998. Acesso em $02 / 07 / 2015$

BRANCALION, P. H.S ; NOVEMBRE, A. D. L.C.; RODRIGUES, R. R. Temperatura ótima de germinação de sementes de espécies arbóreas brasileiras. Revista Brasileira de Sementes, Londrina, vol. $32, n^{\circ} 4$, p. $015-021,2010$.

CARVALHO, P.E. R. Espécies arbóreas brasileiras. Brasília: Embrapa Informação Tecnológica, v.1, 2003. $1039 \mathrm{p}$.

CARVALHO, L.R.; SILVA, E.A.A.; DAVIDE, A.C. Classificação de sementes florestais quanto ao comportamento no armazenamento. Revista Brasileira de Sementes, Pelotas, v. 28, n. 2, p.15- 25, 2006.

FANTI, S. C.; PEREZ, S. C. J. G. A. Processo germinativo de sementes de paineira sob estresses hídrico e salino. Pesquisa Agropecuária Brasileira, Brasília, v.39, n.9, p.903-909, set. 2004.

FANTI, S. C.; PEREZ, S. C. J. G. A. Efeitos do envelhecimento precoce no vigor de sementes de Chorisia speciosa - Bombacaceae. Revista Árvore, Viçosa, v. 29, n. 3, p. 345-352, 2005.

FERREIRA, F.A. Patologia florestal: principais doenças florestais no Brasil. Viçosa: Sociedade de Investigações Florestais, 1989. 570 p. 12.

FIUMARO, R.J. Incidência de fungos e germinação de sementes de paineira. 2010. Trabalho de Conclusão de Curso (Graduação- Engenharia Agronômica). Faculdade Dr. Francisco Maeda. Fundação Educacional de Ituverava.

GOULART, A.C.P. Fungos em Sementes de Soja Detecção, Importância e Controle. EMBRAPA Agropecuária Oeste: Dourados, 2004.72p.

GUEDES, R.S; ALVES, E.U. Substratos e temperaturas para a germinação de sementes de Chorisia glaziovii. Cerne, Lavras, v.17, n.4, p. 525-531, 2011.

LAZAROTTO, M., MUNIZ, M.F.B., SANTOS, A.F. Detecção, transmissão, patogenicidade e controle químico de fungos em sementes de paineira (Ceiba speciosa) Summa Phytopathol., Botucatu, v. 36, n. 2, p. 134-139, 2010.

LEMES, E.Q.; LOPES, J.C. Temperaturas cardinais para germinação de sementes e desenvolvimento de plântulas de paineira Scientia Forestalis, Piracicaba, v. 40, n. 94, p. 179-186, 2012.

LINK, O.; COSTA, E. C. Alguns problemas fitossanitários em viveiros de essências florestais no Rio Grande do Sul. In: SEMINÁRIO SOBRE ATUALIDADES E PERSPECTIVAS FLORESTAIS, 1982, Curitiba. Anais... Curitiba: EMBRAPA, 1982. 265p. 
LORENZI, H. Árvores brasileiras. Nova Odessa: Plantarum, 1992. 352p.

MACHADO, J.C. Patologia de Sementes: fundamentos e aplicações. Lavras:ESAL/FAEPE, 1988.

MARTINS, C.C.; MARTINELLI-SENEME, A., NAKAGAWA, J. Estágio de colheita e substrato para o teste de germinação de sementes de ipê (Tabebuia chrysotricha (Mart. ex DC.) Standl.) Revista Árvore, Viçosa, v.32, n.1, 2008.

MARTINELLI-SENEME, A. M., POSSAMAI, E., RUARO, L.VANZOLINI, S. Germinação e sanidade de sementes de Bauhinia variegata. Revista Árvore, Viçosa, v. 30, n. 5, p.719-724, 2006.

MARTINELLI-SENEME, A.; HOFFMAN, S.; POSSAMAI, E. Colheita e germinação de sementes de ipê (Tabebuia chrysotricha) Scientia Agraria, Curitiba, v.9, n.4 , 419-423, 2008.

MEDEIROS, J. G. F.et al. Qualidade sanitária de sementes de Caesalpinia ferrea: incidência de fungos, controle e efeitos na qualidade fisiológica com o uso de extratos vegetais. Floresta, Curitiba, v. 45 , n. 1, p. 1, 2015.

MENTEN, J.O.M. Prejuízos causados por patógenos associados às sementes. In: Menten, J.O.M. (Ed.) Patógenos em sementes: detecção, danos e controle químico. Piracicaba ESALQ/FEALQ. 1991. p.115135.

NAKAGAWA, J. Testes de vigor baseados no desempenho de plântulas. In: KRZYZANOWSKI, F. C.; VIEIRA, R. D.; FRANÇA NETO, J. B. (Eds.). Vigor de sementes: conceitos e testes. Londrina: ABRATES, 1999. p.1-21

SILVA, R.T.V.et al. Tratamento de sementes e armazenamento na sanidade de sementes de paineira (Chorisia speciosa St. Hil) Semina: Ciências Agrárias, Londrina, v. 24, n. 2, p. 255-260, 2003. 